\title{
Formulation and In-Vitro Evaluation of Gastroretentive Drug Delivery System Containing Ofloxacin
}

\author{
Gaikwad V. D. ${ }^{1}$, Gaikwad M. D. ${ }^{2}$, Yadav P. B. ${ }^{3}$ \\ ${ }^{I}$ (Arvind Gavali College Of Pharmacy, Department of Pharmaceutics, Jaitapur, Satara-415004. India) \\ ${ }^{2}$ (D. Y. Patil College Of Art, Commerce, Science, Department of Biotechnology, Pimpari, Pune-411018. India) \\ ${ }^{3}$ (D. Y. Patil College Of Pharmacy, Department of Pharmaceutical Chemistry, Pimpari, Pune-411018. India)
}

\begin{abstract}
Sustained release gastroretentive dosage forms enable prolonged and continuous input of the drug to the upper parts of the gastrointestinal (GI) tract and improve the bioavailability of medications that are characterized by a narrow absorption window. A new strategy is proposed for the development of gastroretentive dosage forms for ofloxacin preferably once daily. The design of the delivery system was based on the sustained release formulation, with floating and swelling features in order to prolong the gastric retention time of the drug delivery systems. Different polymers, such as psyllium husk, HPMC K100M, crospovidone and its combinations were tried in order to get the desired sustained release profile over a period of $24 \mathrm{~h}$. various formulations were evaluated for buoyancy lag time, duration of buoyancy, dimensional stability, drug content and in vitro drug release profile. It was found that dimensional stability of the formulation increases with the increasing psyllium husk concentration. It was also found that in vitro drug release rate increased with increasing amount of crospovidone due to the increased water uptake, and hence increased driving force for drug release. The optimized formulation was subjected to stability studies at different temperature and humidity conditions as per ICH guidelines.
\end{abstract}

Keywords: Ofloxacin; Sustained release; Psyllium husk; Gastroretentive

\section{Introduction}

Oral sustained release (SR)-dosage forms (DFs) have been developed for the past three decades due to their considerable therapeutic advantages [1]. However, this approach has not been suitable for a variety of important drugs, characterized by a narrow absorption window in the upper part of the gastrointestinal tract, i.e. stomach and small intestine. This is due to the relatively short transit time of the DF in these anatomical segments. Thus, after only a short period of less than $6 \mathrm{~h}$, the SR-DF has already left the upper gastrointestinal tract and the drug is released in non absorbing distal segments of the gastrointestinal tract. This results in a short absorption phase that is often accompanied by lesser bioavailability.

It was suggested that compounding narrow absorption window drugs in a unique pharmaceutical DF with gastroretentive properties would enable an extended absorption phase of these drugs. After oral administration, such a DF would be retained in the stomach and release the drug there in a sustained manner, so that the drug could be supplied continuously to its absorption sites in the upper gastrointestinal tract. This mode of administration would best achieve the known pharmacokinetic and pharmacodynamic advantages of SR-DFs for these drugs [2].

The need for gastroretentive dosage forms (GRDFs) has led to extensive efforts in both academia and industry towards the development of such drug delivery systems [3]. These efforts resulted in GRDFs that were designed in large part based on the following approaches: (a) low density form of the DF that causes buoyancy above gastric fluid [4]; (b) high density DF that is retained in the bottom of the stomach; (c) bioadhesion to the stomach mucosa [5]; (d) slowed motility of the gastrointestinal tract by concomitant administration of drugs or pharmaceutical excipients [6]; (e) expansion by swelling or unfolding to a large size which limits emptying of the DF through the pyloric sphincter [7].

The objective of present work was to develop gastroretentive formulation, which releases drug in the stomach and upper gastrointestinal (GI) tract, and form an enhanced opportunity of absorption in the stomach and upper GI tract rather than the lower portions of the GI tract. Example of substance whose bioavailability is strongly dependent on the local physiology in the GI tract and which preferably is absorbed in the higher sections of the intestine is ofloxacin. Ofloxacin is readily soluble in the acidic environment of the stomach. In the intestine, where neutral to slightly alkaline $\mathrm{pH}$ conditions prevail; however, precipitation of the active compound occurs, which adversely affects absorption in the lower sections of the intestine. There is a need for systems that reside in the stomach over a relatively long time and release the active compound there in a sustained manner [8]. This necessitated the design and development of sustained release gastroretentive drug delivery system for ofloxacin using suitable polymers. 


\section{Materials And Methods}

Ofloxacin and psyllium husk were gifted by Macleoid Pharmaceuticals, India. HPMC K100M, PVP K30 and crospovidone were obtained as gift samples from Torrent pharmaceutical, Ahmedabad. Talc and magnesium stearate were gifted by M/s Bayer India Ltd., India. All other solvents and reagents were purchased from Ranbaxy chemicals, India, and were of analytical grade.

Typical sustained release formulations of ofloxacin are listed in Tables 1 and 2. Tablets were made by using psyllium husk (gelling agent), HPMC K100M (hydrophilic polymer), crospovidone (swelling agent), sodium bicarbonate (gas-generating agent) and betacyclodextrin (channeling agent). Tablets were made by using wet granulation process with PVP K30 (5\%, w/v, isopropyl alcohol). Tablets were compressed on RIMEK multi station punching machine using $12.5 \mathrm{~mm}$ flat punches.

Table 1: Formulation composition to study the effect of psyllium husk and HPMC K100M on in vitro release of ofloxacin

\begin{tabular}{|l|l|l|l|l|l|}
\hline Composition (mg/tablet) & F1 & F2 & F3 & F4 & F5 \\
\hline Ofloxacin & 400 & 400 & 400 & 400 & 400 \\
\hline Psyllium husk & 75 & 100 & 125 & 100 & 100 \\
\hline HPMC K100M & 40 & 40 & 40 & 30 & 50 \\
\hline Sodium bicarbonate & 70 & 70 & 70 & 70 & 70 \\
\hline Crospovidone & 200 & 200 & 200 & 200 & 200 \\
\hline PVP K30 (5\% in IPA) & 20 & 20 & 20 & 20 & 20 \\
\hline
\end{tabular}

Table 2: Formulation composition to study the effect of crospovidone, sodium bicarbonate and betacyclodextrin on in vitro release of ofloxacin

\begin{tabular}{|l|l|l|l|l|l|l|}
\hline $\begin{array}{l}\text { Composition } \\
\text { (mg/tablet) }\end{array}$ & F6 & F7 & F8 & F9 & F10 & F11 \\
\hline Ofloxacin & 400 & 400 & 400 & 400 & 400 & 400 \\
\hline Psyllium husk & 100 & 100 & 100 & 100 & 100 & 100 \\
\hline HPMC K100M & 40 & 40 & 40 & 40 & 40 & 40 \\
\hline Sodium bicarbonate & 70 & 70 & 60 & 80 & 70 & 70 \\
\hline Crospovidone & 0 & 100 & 200 & 200 & 200 & 200 \\
\hline PVP K30 (5\% in IPA) & 20 & 20 & 20 & 20 & 20 & 20 \\
\hline Betacyclodextrin & - & - & - & - & 50 & 100 \\
\hline
\end{tabular}

\subsection{In vitro release study}

The release of ofloxacin from the tablets was studied using USP dissolution Apparatus I. The dissolution medium was phosphate buffer $\mathrm{pH} 1.2$ for first $2 \mathrm{~h}$, phosphate buffer $\mathrm{pH} 4.5$ for next $2 \mathrm{~h}$ and $\mathrm{pH} 7.4$ for remaining hours [9], the volume being $900 \mathrm{ml}$. The temperature was maintained at $37 \pm 0.5{ }^{\circ} \mathrm{C}$. The rotation speed was $100 \mathrm{rpm}$. Five milliliters of aliquot were withdrawn at predetermined time intervals of 1, 2, 3, 4, 6, 8, $10,12,14,16$ and $24 \mathrm{~h}$. The medium was replenished with $5 \mathrm{ml}$ of fresh buffer each time. Sample was analyzed by using UV spectrophotometer at $291 \mathrm{~nm}$.

\subsection{Buoyancy lag time and the duration of buoyancy}

The buoyancy lag time and the duration of buoyancy were determined in the USP dissolution Apparatus II in an acid environment. The time interval between the introduction of the tablet into the dissolution medium and its buoyancy to the top of dissolution medium was taken as buoyancy lag time and the duration of buoyancy was observed visually [10].

\subsection{Dimensional stability}

The dimensional stability of the formulations was studied using USP dissolution Apparatus II. The dissolution medium was phosphate buffer $\mathrm{pH} 1.2$ for first $2 \mathrm{~h}$, phosphate buffer $\mathrm{pH} 4.5$ for next $2 \mathrm{~h}$ and $\mathrm{pH} 7.4$ for remaining hours, and the volume being $900 \mathrm{ml}$. The temperature was maintained at $37 \pm 0.5{ }^{\circ} \mathrm{C}$. The rotation speed was $50 \mathrm{rpm}$. The dimensional stability of ofloxacin formulations was observed visually.

\section{Results And Discussion}

\subsection{Effect of psyllium husk on in vitro drug release and integrity of formulations}

Effect of different concentrations of psyllium husk on in vitro release of ofloxacin was studied. Initially, tablet containing $75 \mathrm{mg}$ of psyllium husk (F1) could not retain its physical integrity for desired period (24 h) of time. As the concentration of psyllium husk increases (F2 and F3), it retains integrity up to desired 
period of time $(24 \mathrm{~h})$. It is important to maintain the physical integrity of the tablet in case of once daily formulations. If it does not maintain its physical integrity, tablet could be broken down into smaller fragments and escape from the upper part of the GI tract. Hence, an attempt was made in order to increase the physical integrity of the tablets using psyllium husk. Further, formulation (F1) provided higher burst drug release as compared to the marketed formulation as shown in Fig. 1. In case of F1 and marketed formulations, burst drug release after $2 \mathrm{~h}$ was $34.06 \pm 0.52$ and $31.08 \pm 0.89 \%$, respectively. Therefore, amount of psyllium husk was increased to $100(\mathrm{~F} 2)$ and $125 \mathrm{mg} /$ tablet (F3). As the concentration of psyllium husk increases, initial burst drug release as well as drug release in the latter hours decreases as compared to the marketed formulation. In case of $\mathrm{F} 2$ and F3 formulations, burst drug release after $2 \mathrm{~h}$ was found to be $28.22 \pm 0.96$ and $24.22 \pm 1.28 \%$, respectively. In case of formulation F1 and F3, cumulative drug release at the end of $24 \mathrm{~h}$ was found to be $53.60 \pm 1.86$ and $47.20 \pm 1.74 \%$, respectively. This might be due to the gelling properties of psyllium husk. Psyllium husk forms thick gel at higher concentrations, which could have contributed to the decrease in drug release. Drug associated with the surface of tablet matrix could have also contributed to the initial burst release. As the surface associated drug was released, psyllium husk matrix could have contributed to the slower drug release over a period of $24 \mathrm{~h}$. Formulation (F2) containing $100 \mathrm{mg}$ of psyllium husk maintained its physical integrity for $24 \mathrm{~h}$ and showed similar pattern of drug release as compared to the marketed formulation, hence, selected for the further studies.

Ofloxacin is soluble in aqueous solution with $\mathrm{pH}$ between 2 and 5 . It is sparingly to slightly soluble in aqueous solution with $\mathrm{pH}$ 7. Hence, developed formulations as well as marketed formulation could not release total amount of the drug into the dissolution medium by $\mathrm{pH}$ change method.

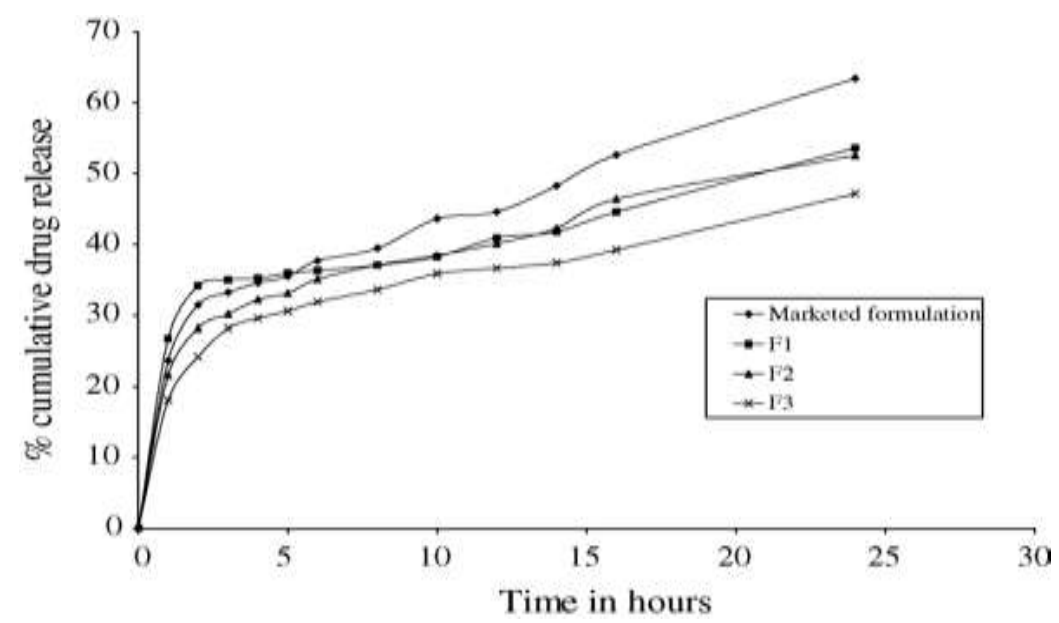

Fig.1. Effect of psyllium husk concentration on in vitro drug release of ofloxacin $(n=6)$. Standard deviation was found to be less than $2 \%$ in all the in vitro drug release profiles.

\subsection{Effect of HPMC K100M on in vitro release of ofloxacin}

Initially, HPMC K100M was tried in concentration of $30 \mathrm{mg} /$ tablet (F4). The formulation provided higher burst drug release as compared to marketed formulation as shown in Fig. 2. In case of F4 and marketed formulations, burst drug release after $2 \mathrm{~h}$ was found to be $34.08 \pm 0.92$ and $31.08 \pm 0.89 \%$, respectively.

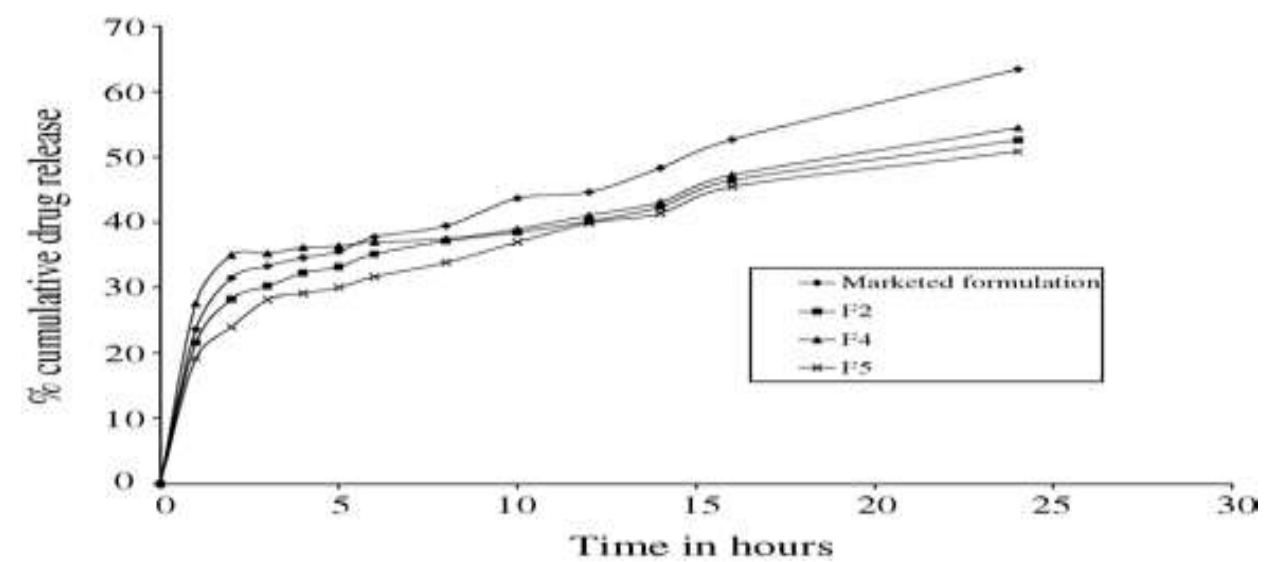

Fig.2. Effect of HPMC K100M concentration on in vitro drug release of ofloxacin $(n=6)$. Standard deviation was found to be less than $2 \%$ in all the in vitro drug release profiles. 
Therefore, amount of HPMC K100M was increased to $40 \mathrm{mg} / \mathrm{tablet}$ (F2), the formulation provided burst drug release comparable to the marketed formulation. Further increase in amount of HPMCK100M to $50 \mathrm{mg}$ (F5) provided low burst drug release as compared to the marketed tablets. Formulation (F5) showed $24.01 \pm 1.23 \%$ of burst drug release at the end of $2 \mathrm{~h}$. High HPMC K100M content results in a greater amount of gel being formed. This gel increases diffusion path length of the drug. Its viscous nature also affects diffusion coefficient of the drug. As a result, reduction in drug release is obtained. Thus, $40 \mathrm{mg}$ (F2) HPMC K100M was selected for further studies.

\subsection{Effect of crospovidone on in vitro release of ofloxacin}

In order to improve the release profile of the formulations, concentration of crospovidone was increased. Crospovidone acts as a swelling agent, which is capable of swelling greater than its original volume and preferably to at least twice its original volume when coming into contact with an aqueous fluid, such as gastrointestinal fluid. The swelling agent, which normally swells to several times its original volume in water, exhibits a controlled swelling in the presence of water soluble hydrophilic polymers [11]. Three concentrations 0mg (F6), $100 \mathrm{mg}$ (F7) and $200 \mathrm{mg}$ (F2) of crospovidone were tried. As the concentrations of crospovidone increases from 0 to $200 \mathrm{mg} /$ tablet, drug release has increased as shown in Fig. 3. Formulation (F6), which does not contain crospovidone, showed $38.71 \pm 1.64 \%$ of cumulative drug release at the end of $24 \mathrm{~h}$. Formulation containing $100 \mathrm{mg}$ (F7) showed $49.64 \pm 1.32 \%$ cumulative drug release whereas formulation containing $200 \mathrm{mg}$ (F2) showed 52.55 $\pm 1.02 \%$ cumulative release at the end of $24 \mathrm{~h}$. As the concentration of crospovidone increases, water uptake capacity of the formulation increases (data not shown). This increases the porosity of the matrix, which results in increased drug release from the matrix system.

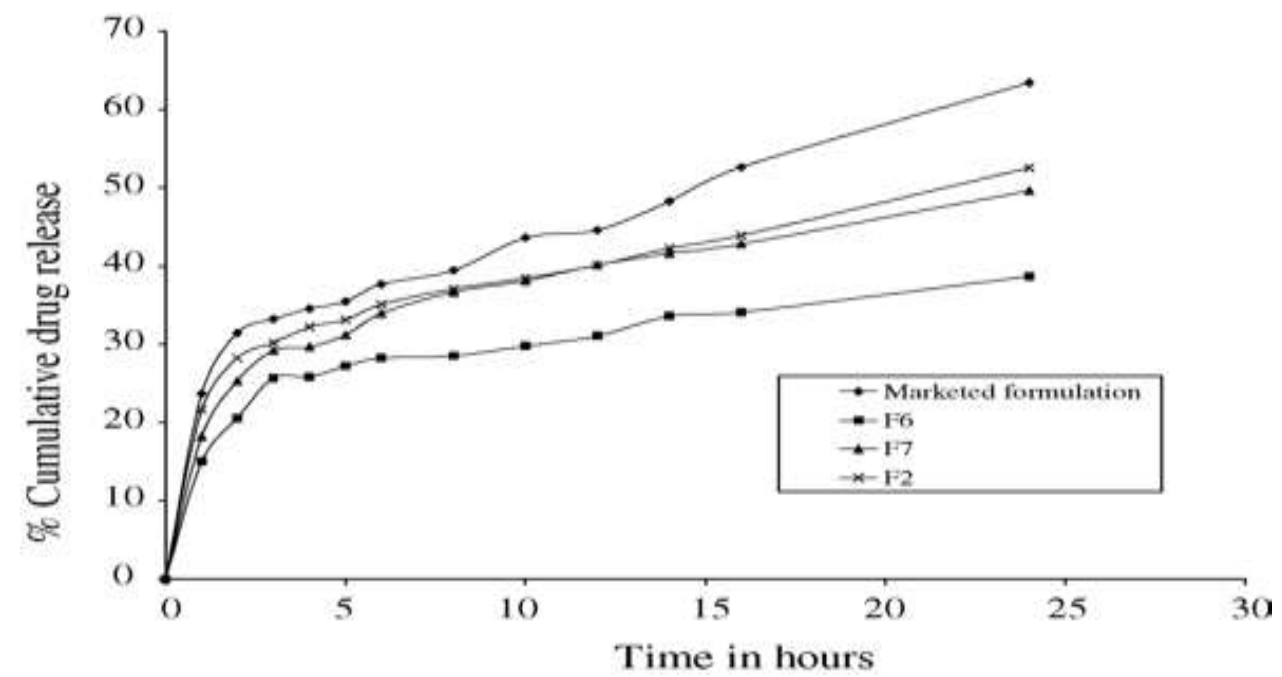

Fig.3. Effect of crospovidone concentration on in vitro drug release of ofloxacin $(n=6)$. Standard deviation was found to be less than $2 \%$ in all the in vitro drug release profiles.

\subsection{Effect of gas-generating agent on in vitro release of ofloxacin}

As the concentration of sodium bicarbonate increases from 60 to $80 \mathrm{mg} /$ tablet, drug release decreases as shown in Fig. 4. This might be due to the alkaline nature of sodium bicarbonate. Sodium bicarbonate creates an alkaline environment around the tablet. Ofloxacin is less soluble in alkaline environment that decreases the drug release from the formulation. Formulation containing $60 \mathrm{mg}$ sodium bicarbonate (F8) showed $54.91 \pm 1.30 \%$ cumulative drug release whereas formulation containing $80 \mathrm{mg}$ of sodium bicarbonate (F9) showed $47.20 \pm 1.35 \%$ cumulative drug release at the end of $24 \mathrm{~h}$. Formulation containing $70 \mathrm{mg}$ sodium bicarbonate (F2) showed $52.55 \pm 1.02 \%$ cumulative drug release at the end of $24 \mathrm{~h}$.

In such systems, sodium bicarbonate acts as a gas generating agent. It generates gas when it comes into contact with an acidic environment of the stomach. This gas entraps into the matrix of water-soluble polymers and the formulation floats in an acidic environment of the stomach.

As the concentration of sodium bicarbonate increases from 60 (F8) to $80 \mathrm{mg} /$ tablet (F9), floating lag time has reduced to $25 \mathrm{~s}$. As the concentration of sodium bicarbonate increases from 60 to $80 \mathrm{mg} /$ tablet, duration of floating has also been increased from 18 to $24 \mathrm{~h}$ as shown in Table 3 . This might be due to the gas generated by $60 \mathrm{mg}$ sodium bicarbonate might not be sufficient to keep formulation floating for prolonged period of time whereas in case of gas generated by $80 \mathrm{mg}$ sodium bicarbonate was sufficient to keep formulation floating for $24 \mathrm{~h}$. 
Table 3: Effect of sodium bicarbonate concentration on onset and duration of floating

\begin{tabular}{|c|c|c|}
\hline Amount of sodium bicarbonate (mg) & Onset of floating (s) & Duration of floating (h) \\
\hline 60 & $40 \pm 3$ & 18 \\
\hline 70 & $30 \pm 4$ & 24 \\
\hline 80 & $25 \pm 3$ & 24 \\
\hline
\end{tabular}

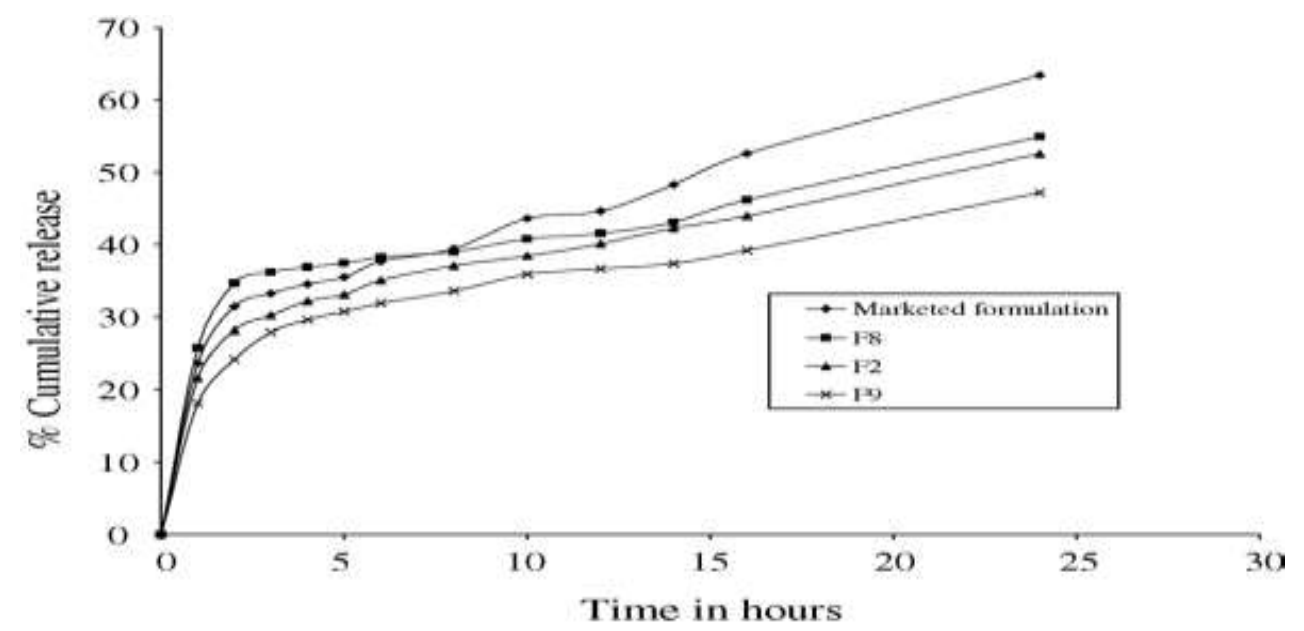

Fig.4. Effect of sodium bicarbonate concentration on in vitro drug release of ofloxacin $(n=6)$. Standard deviation was found to be less than $2 \%$ in all the in vitro drug release profiles.

\subsection{Effect of betacyclodextrin on in vitro release of ofloxacin}

In order to improve the drug release profile from the formulation, betacyclodextrin was added in to the formulation. As the concentration of betacyclodextrin increases from 0 to $100 \mathrm{mg} / \mathrm{tablet}$, initial burst releases as well as drug release in the latter hours have been increased as shown in Fig. 5. In case of formulation containing 0mg (F2) and $50 \mathrm{mg}$ betacyclodextrin (F10), cumulative drug release after $24 \mathrm{~h}$ was $52.55 \pm 1.02$ and $57.90 \pm 1.32 \%$, respectively. The formulation containing $100 \mathrm{mg}$ betacyclodextrin/tablet (F11) showed similar drug release as that of marketed formulation. Betacyclodextrin dissolves rapidly from the tablet matrix into the medium and it creates porosity to the matrix, which results in increase in drug release from the tablet matrix. In vitro drug release studies were also carried out in $\mathrm{pH} 1.2$ phosphate buffer, developed as well as marketed formulations released the total amount of the drug in $\mathrm{pH} 1.2$ phosphate buffer for $24 \mathrm{~h}$ (data not shown).

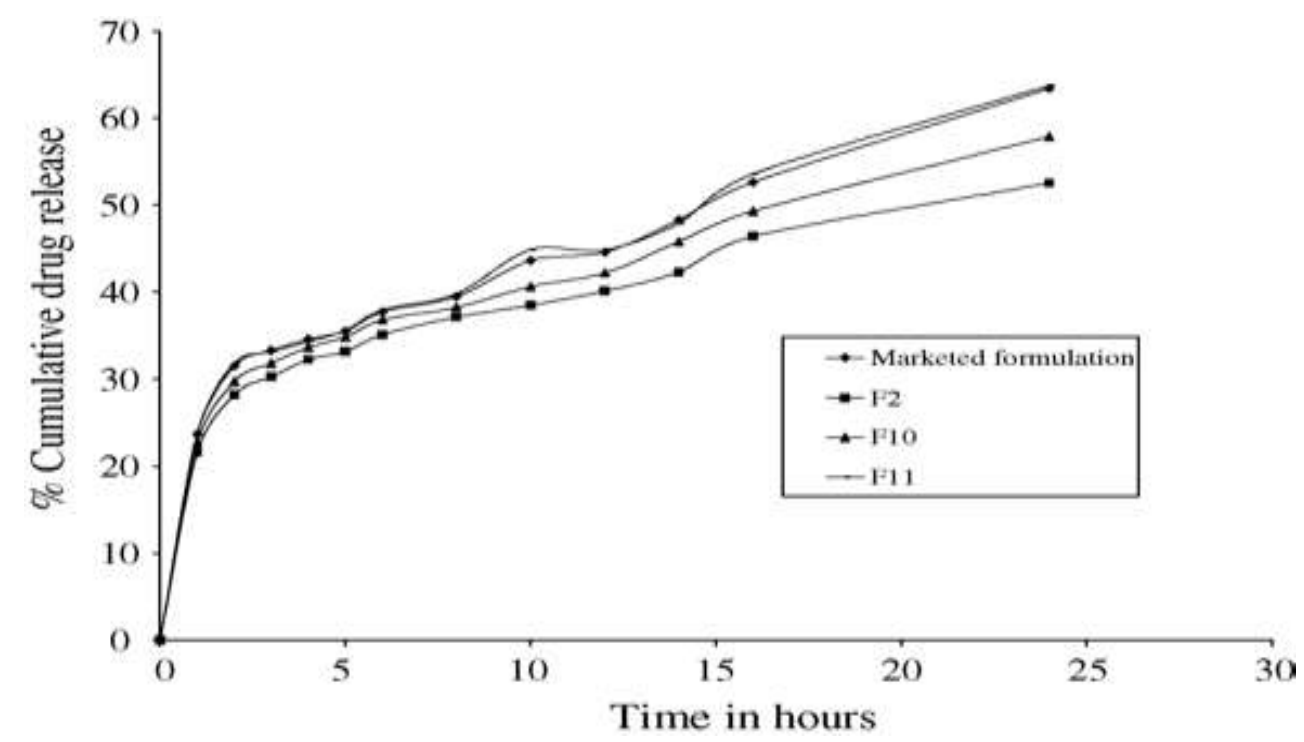

Fig.5. Effect of betacyclodextrin concentration on in vitro drug release of ofloxacin $(n=6)$. Standard deviation was found to be less than $2 \%$ in all the in vitro drug release profiles. 


\section{Conclusion}

We conclude that psyllium husk and HPMCK100M increases the dimensional stability of the formulations, which is necessary in case of once daily formulations. Sodium bicarbonate acts as a gasgenerating agent, which is necessary in case of gastroretentive dosage forms. Crospovidone improved the drug release profile and swelling factor of psyllium husk based formulations. We also conclude that channeling agents, such as betacyclodextrin are useful to increase the initial burst release from psyllium husk based formulations. The optimized formulation was found to be stable at all the stability conditions.

\section{Acknowledgements}

We are thankful to Macleoid Pharmaceuticals, India and Torrent pharmaceutical, Ahmedabad, for providing us gift samples of drug and polymers. We are also thankful to department of pharmaceutics, Arvind Gavali College of Pharmacy, Satara.

\section{References:}

[1] A. Hoffman, Pharmacodynamic aspects of sustained release preparations. Adv. Drug Deliv. Rev. 33, $1998,185-199$.

[2] S. J. Hwang, H. Park, K. Park, Gastric retentive drug-delivery systems. Crit. Rev. Ther. Drug Carrier Syst. 15, 1998, $243-284$.

[3] A. A. Deshpande, N.H. Shah, C.T. Rhodes, W. Malick, Controlled-release drug delivery systems for prolonged gastric residence: an overview. Drug Dev. Ind. Pharm. 22, 1996, 531-539.

[4] B. N. Singh, H.N. Kim, Floating drug delivery systems: an approach to oral controlled drug delivery via gastric retention. J. Control. Release 63, 2000, 235-259.

[5] A. J. Moes, Gastroretentive dosage forms. Crit. Rev. Ther. Drug Carrier Syst. 10, 1993, 143-195.

[6] A. Rubinstein, D. R. Friend, A. J. Domb, (Ed.), Polymeric Site-Specific Pharmacotherapy.Wiley, Chichester, pp. 1994, $267-313$.

[7] R. C. Mamjek, E. S. Moyer, Drug Dispensing Device and Method. U.S. Patent. 4, 1980, $207,890$.

[8] H. Sen, R. S. Kshirsagar, Pharmaceutical composition for a controlled release of active ingredient. WO024187, 2002.

[9] Y. Wang, Cephalexin could be formulated as sustained release dosage forms. J. Pharm. Sci. 67, 1978, 1620-1622.

[10] L. Yang, J. Eshraghi, R. Fassihi, A new intragastric delivery system for the treatment of Helicobacter pylori associated gastric ulcer: in vitro evaluation. J. Control. Release 57, 1999, 215-222.

[11] N. Talwar, J. Staniforth, Orally Administered Controlled Delivery System for Once Daily Administration of Ciprofloxacin. WO $01 / 64183,2001$ 